\title{
Role of Psychology in Interventional Pain Management
}

\section{To the editor:}

I am writing regarding two articles in the April 2002 issue.

i. Manchikanti et al (1) "Psychological Status in Chronic Low Back Pain" is in agreement with many previous publications as the authors point out. What is most important is the apparent utility of the P3 test as a tool for rapidly and affordably assessing the chronic pain patient, and perhaps the acute/subacute pain patient. The correlation with MMPI was encouraging in further validating the $\mathrm{P} 3$ test to those more familiar with MMPI. I look forward to an article that assesses recent onset radiculopathy patients and follows them through treatment with pre and post treatment P3 testing and correlation to outcome, as one would predict from Von Korff et al's (2) study. The title of the article might have been more accurately reflective of content as "Psychological Status in Chronic Low Back Pain as Assessed by the P3 test; a useful tool for interventional pain physicians.”

The method section states that the control group was screened to exclude any subjects with prior psychological history. This will obviously select a skewed population that one would expect to test with close to zero positive results. Yet the chronic lumbar pain patients were not excluded for psychological diagnoses or treatment that predated the onset of their pain, which would be a hard condition to assess. A better control would randomly pick control participants with no lumbar or other chronic pain complaint regardless of psych history. Only one variable should differentiate the two groups - pain vs no pain. There is a prevalence of depression, anxiety and somatization in the general population, as the author also mentions. Those persons should not be excluded from the controls.

The prevalence of depression, anxiety and somatization is higher in chronic pain patients. Some of this is presumed reversible with treatment of the pain. We should not exaggerate the difference from the general population by excluding any control group patients with history of psychological diagnoses or treatment. Improvement in P3 scores post treatment should approximate the general population norm, not zero. If we use a falsely lowered general population standard, our treatment outcomes will appear falsely poor.

Will such testing help place individuals into different treatment algorithms? Some patients might avoid unnecessary and expensive psychological assessment and treatments in practices that routinely run all patients through both physical and psychological treatment. For those practices that routinely do not include psychological co-treatment, the test may help to early on identify individuals who would be better treated by or along with the psychologist.

Overall, I found the article informative with excellent references. It promotes a less cumbersome way to assess the psychological status of patients. I foresee this could be an important tool for interventional pain practices. Many pain clinics have found the psychology costs outweigh revenue. The P3 may help preserve and promote psychological assessment of chronic pain patients.

ii. In "Do Number of Pain Conditions Influence Emotional status" by Manchikanti et al (3), the same control group comment is valid.

The fact that multiple pain complaints are associated with more anxiety, depression and bodily pre-occupation should surprise no one. Negative factors are logically additive as are positives in any situation, medical or not. In the non-pain medical realm multi-system medical problems are associated with depression and poor outcome. The individual with multiple negative stress factors such as spousal death, job loss and major illness, and those with substance or childhood or sexual abuse are at higher risk of suicide than one without such risk factors (4-7).

Why has nature coupled psychological distress to physical distress? Are the minds of chronically ill patients telling them to be concerned enough to take action? Perhaps, from a Darwinian perspective they realize that they are less capable of competing for survival. Does depression prepare us to whither and die, or to help our predators survive on the infirm to favor evolution of the strong? Is societal permission to suffer, express suffering and to be 
dependent on others encouraging the prevalence of anxiety, depression and somatization? Do multiple bodily dysfunctions directly alter the neurotransmitter and receptor balance in an adverse manner? The present study and others like it tell us that psychological dysfunction occurs, but not why.

What works best - to chemically alter mood in order to improve pain, or to alter pain to improve mood? We have all appreciated that both can be effective. How do we use this knowledge to better treat our patients? First, interventionalists must accept that their patients do have psychological comorbidities.

Joseph F. Jasper, MD

Advanced Pain Medicine Physicians

Tacoma, WA 98465-1613

http://www.tacoma-spine-injection.com

\section{REFERENCES}

1. Manchikanti L, Pampati V, Damron K et al. Evaluation of psychological status in chronic low back pain: Comparison with general population. Pain Physician 2002; 5:149-155.

2. Von Korff M, Deyo RA, Cherkin D et al. Back pain in primary care. Outcomes at 1 year. Spine 1993; 18:855-862.

3. Manchikanti L, Pampati V, Beyer C et al. Do number of pain conditions influence emotional status? Pain Physician 2002; 5:200-205.

4. Plunkett A, O’Toole B, Swanston H et al. Suicide risk following child sexual abuse. Ambul Pediatr 2001; 1:262-266.

5. Szanto K, Gildengers A, Mulsant BH, et al. Identification of suicidal ideation and prevention of suicidal behavior in the elderly. Drugs Aging 2002; 19:11-24.

6. Ogedegbe G, Charlson M, Allegrante J. Determinants of post-operative depression after coronary angioplasty. J Clin Epidemiol 2002; 55:628.

7. Granier E, Boulenger JP. Suicidal Risk Scale. Encephale 2002; 28:29-38. 